motoneurones and that facilitatory fibres to the flexor motoneurones may exsert reciprocal inhibitory effect upon extensor motoneurones.

Neither galvanic skin response nor change in blood pressure by pyramid stimulation was elicited in this present experiment.

\title{
20. An Experimental Neuropathological Study of the Effects of High-Frequency Focused Ultrasound on the Brain of Animals
}

\author{
Hiroshi Hasegawa, Kijuro Mitsuma, Masao Watanabe, Yoshiaki Sugiyama, \\ Keizo KaWaKami and Komei UeKI \\ Department of Neurosurgery, Niigata University
}

Well localized lesions were produced in the brain of cats and rabbits by means of high intensity focused ultrasound. Animals wcre allowed to survive for verying periods, from 3 hours to 78 days following irradiation. The focused ultrasound was generated by concave titanic acid barium transducer. Frequency, 1.1 $\mathrm{MC}$, focal lengtht, $7 \mathrm{~cm}$, diameter of generator, $6 \mathrm{~cm}$ and peak intensity was 1551 $\mathrm{w} / \mathrm{cm}^{2}$. The large ultrasound lesions were characterized by a double zonation, but small lesions showed no zonation. 3 hours after irradiation the pathologic changes in the ultrasound lesions were loosening of tissue, pale and fragmented myelin sheets and pyknosis of nucleus of glia. The borderline of lesion was well defined. The inflammatory changes were pronounced 3 days after irradiation. The numerous macrophages came together at peripheral portion of lesions, and they entered into the lesions. 3 weeks after irradiation the lesions were occupied by macrophages, fibrocytes and lymphocytes. At this stage macrophages were swollen and contained small fragments of myelin in them. Astrocytes showed necrobiosis and proliferation of fibrous tissues and blood vessels were seen. 78 days after irradiation the lesions were surrounded by connective tissue and they were well localized. But the most part of the lesion was occupied by regularly arranged macrophages and scar did not yet developed. There was no marked change in size of lesions during the period of 3 hours to 78 days, there were never any late changes comparable to those following $\mathrm{X}$-radiation. There was no change along the pathway of the ultrasound beam. Blood brain barrier was also destructed in the lesions and its reconstruction was completed after about 1 month. 